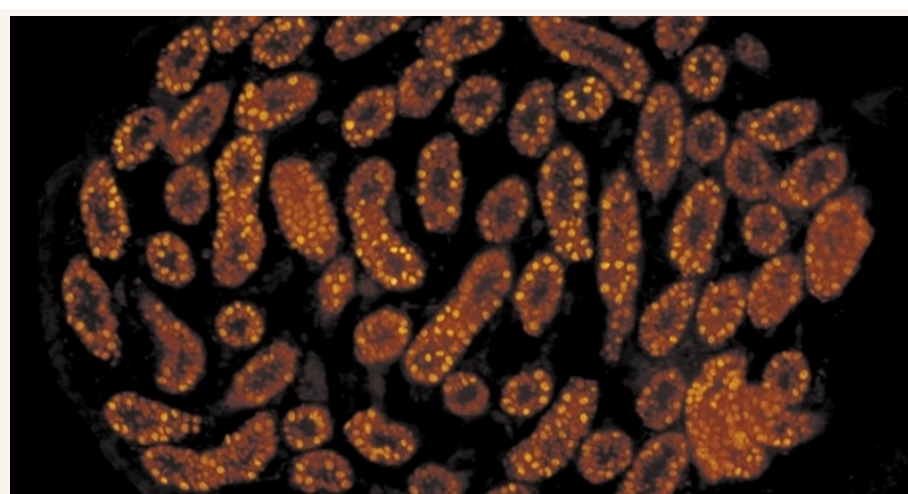

Very strong expression of Dmrt1 protein in spermatogonia and more uniform expression in Sertoli cells. Courtesy of David Zarkower.

\section{SEX DETERMINATION}

\section{It's a guy thing}

Until recently, there seemed to be little in common between the sexdetermining pathways among the animal phyla. However, there is now functional data to indicate that a transcription factor may be a regulator of male development that is shared by vertebrates, flies and worms.

So far, the only known similarities between genes involved in sexual development are between the mab-3 gene in Caernorhabditis elegans and the doublesex ( $d s x$ ) gene of Drosophila melanogaster. Both genes encode transcriptional regulators that have a conserved zinc-finger-like DNA-binding domain, called the DM domain. The two proteins are also functionally related, as they are both required for differentiation of male-specific sense organs, regulation of yolk protein transcription and normal male mating behaviour. A DMdomain gene, Dmrt1, has been implicated in sexual development in vertebrates as well, a fact that prompted Raymond and colleagues to investigate whether it is required specifically for male development in the mouse.

A null allele of Dmrt1 was made by the targeted deletion of the DM domain (and the transcriptional start site). Male mice heterozygous for Dmrt1 appear normal, but homozygous males fail to maintain testis differentiation from postnatal day seven and onwards. The fact that $\mathrm{Dmrt}^{-/-}$females develop into normal, fertile adults, supports the idea that Dmrtl function is male specific.

Dmrt1 is necessary in the male gonad for the survival and differentiation of both somatic and germline cells. Germ cells are missing in the very small testes of the homozygous mutant mice, and the Sertoli cells, which provide nourishment to the early sperm cells, have abnormal cell morphology, overproliferate in early seminiferous tubules, and later die. Whether the germ-cell defect is cell autonomous, or results from the incorrect development of the Sertoli cells, is under investigation. So far, no point mutations have been found in the human DMRT1 gene. But there is a similarity between the defects observed in the Dmrt $1^{-/-}$male mice with those of human males carrying a distal deletion on $9 \mathrm{p}$, where DMRT1 is located. This indicates that DMRT1 - alone or in combination with one or more tightly linked genes might be required for testis development in humans. $S R Y$, the testis-determining gene, was discovered around ten years ago. When it emerged that SRY was exclusive to mammals, researchers began to look for its downstream targets in the hope that, unlike SRY, it would be common to all vertebrates. Perhaps the identification of Dmrt1 and this new data on its evolutionary conservation relating to a maledetermining function in vertebrates, flies and worms, may mean that this hope has been realized.

Many developmental processes such as body-axis establishment or segmentation, have a long-standing reputation for being evolutionarily conserved between phyla. The discovery that a certain protein signature (the DM domain) can help define male versus female development may mean that sex determination can now be added to this list.

Tanita Casci

(2) References and links ORIGINAL RESEARCH PAPER Raymond, C. S. et al. Dmit1, a gene related to worm and fly sexual regulators, is required for mammalian testes differentiation. Genes Dev. 14 2587-2595 (2000) WEB SITE David Zarkower's lab
H I G H L I G H T S

\section{IN BRIEF}

\section{GENE EXPRESSION}

Evidence that processed small dsRNAs may mediate sequence-specific mRNA degradation during RNAi in Drosophila embryos.

Yang, D. et al. Curr. Biol. 10, 1191-1200 (2000)

RNA interference (RNAi) is a process by which an introduced double-stranded ( $d s$ )RNA specifically silences the expression of genes through degradation of their cognate mRNA. Experiments in vitro indicate that the introduced dsRNA is converted into 21-23 nucleotide 'guide RNAs', which label the target mRNAs to be destroyed by nuclease digestion. Using a quantitative RNAi assay based on measuring luciferase expression in Drosophila melanogaster embryos, the authors show that the 21-23 nucleotide RNAs also mediate RNAi in vivo, with the antisense strand determining substrate specificity.

\section{HUMAN GENETICS}

Small evolutionarily conserved RNA, resembling $\mathrm{C} / \mathrm{D}$ box small nucleolar RNA, is transcribed from PWCR1, a novel imprinted gene in the Prader-Willi deletion region, which is highly expressed in brain.

de los Santos, T. et al. Am. J. Hum. Genet. 67, 1067-1082 (2000)

Paternal inheritance of a deletion in the $15 \mathrm{q} 11-\mathrm{q} 13$ region causes the neurodevelopmental disorder, Prader-Willi syndrome (PWS). Several genes in this region are imprinted and are expressed only from the maternally or paternally derived allele. $P W C R 1$ is such a gene - it encodes an untranslated small nucleolar RNA (snoRNA) that is transcribed only from the paternal allele and is highly expressed in human and mouse brain. Its expression is abolished in PWS brains and its loss might contribute to PWS pathology.

\section{DEVELOPMENTAL BIOLOGY}

\section{Conservation of the sequence and temporal} expression of let-7 heterochronic regulatory RNA.

Pasquinelli, A. E. et al. Nature 408, 86-89 (2000)

In Caenorhabditis elegans, a small 21-nucleotide RNA molecule encoded by the let-7 gene regulates the late developmental transitions in the worm's life cycle. Now it seems that the worm let-7 gene is shared by all the main groups of bilaterally symmetrical animals (including humans), where it is expressed at important developmental transitions, such as before fly metamorphosis. Small RNAs such as let-7 probably function by pairing with target RNAs and preventing their translation. This study also shows that the genetic targets of let-7 have been evolutionarily conserved. 